\title{
A Study on Social Psychology Factors of Golfers in South Korea
}

\author{
Won-ick Song ${ }^{1}$ and Byoungwook $\mathrm{Ahn}^{2 *}$ \\ ${ }^{1}$ Associate Professor, Department of Sport Leisure Industry, Sehan University, Korea \\ ${ }^{2}$ Aisstant Professor, Department of Leisure Marine Sports, Hanseo University, Korea \\ ${ }^{1}$ swi3436@hanmail.net, ${ }^{2}$ bwahn75@hanseo.ac.kr
}

\begin{abstract}
The purpose of study is to investigate on the social psychology factors of golfer in South Korea. The participants in this study were 352 people who live in Seoul, and Gyeonggi-do. For the data analysis in this study confirmatory factor, reliability, and multiple regression analyses were performed. The results were as follows. First, personal factors and structural factors of leisure facilitation had influence on the intrinsic factors of psychological well-being. Second, the individual factors of leisure facilitation had influence on the intrinsic factors of psychological well-being. Third, the individual and structural factors of leisure facilitation had influence on the self-realization of psychological well-being. Fourth, the individual factors of leisure facilitation had influence on the confidence of psychological well-being. So, we found that it is necessary to build an infrastructure to participate in golf as a leisure activity, and the high psychological well-being had an effect on improving the quality of life of individuals.
\end{abstract}

Keywords: Leisure, Golfer, Psychology, Leisure activity, Behavior

\section{Introduction}

\subsection{Purpose of the Study}

According to the Korea Culture and Tourism Research Institute, an increasing percentage of Korean people are participating in leisure activities every year [1]. The people can be attributed to the facts that Koreans can increase their energy levels through leisure participation, and that modern people prefer leisure activities that involve active participation rather than static and unproductive leisure activities. The change in modern people's perceptions of leisure activity participation has had a positive effect on promoting the participation of many people in leisure activities, and it has allowed them to participate in various leisure activities outside of urban areas.

The leisure and psychosocial factors to be identified in this study are leisure facilitation and psychological well-being. First, leisure facilitation is a concept that was first proposed by Raymore [5]. Raymore [5] presented a leisure facilitation model to study the behavior of participants in leisure activities. Leisure facilitation refers to all the facilitating factors that enable individuals to participate in leisure activities [6-7].

The next factor is psychological well-being. Psychological well-being refers to the positive cognitive and emotional states that are integrated in the individual expression of a variety of factors. Many psychologists have examined individual happiness by measuring how individuals rate their lives or how satisfied individuals are with their lives [11-12].

Article history:

Received (December 26, 2020), Review Result (January 26, 2021), Accepted (April 9, 2021) 
Therefore, this study is to investigate how leisure facilitation affects psychological wellbeing among amateur golfers in South Korea. So, we study the causes of the increase in golf participation among various leisure activities in terms of leisure facilitation factors, while also examining the effects on the psychological well-being of amateur golfers.

\subsection{Research hypothesis}

First, the leisure facilitation will influence on intrinsic of psychological well-being. Second, the leisure facilitation will influence on flow of psychological well-being. Third, the leisure facilitation will influence on self-realization of psychological well-being. Forth, the leisure facilitation will influence on confidence of psychological well-being.

\section{Method}

\subsection{Research participants}

The subjects of this study were selected as adult men and women who participate in golf activities and who live in Seoul, Incheon, and Gyeonggi-do. The convenience sampling method was used to select study subjects. The demographic analysis data of the study subjects were as follows: For gender, there were 211 men and 141 women. In terms of age, 89 people were in their 30s or under, 112 people were in their 40s, and 151 people were in their 50s or over. Regarding the participation period, it was under 6 months for 94 people, over 6 months and under 1 year for 98 people, over 1 year and under three years for 105 people, and 3 years or more for 55 people.

\subsection{Measurement}

To measuring leisure facilitation, the questionnaire by Kim and Lee [18] that was developed based on the Raymore model [5] was used. To measure psychological well-being, this study used the questions used in Yang's physical activity and psychological well-being, which were based on Waterman [19]'s Personally Expressive Activities Questionnaire and Ryff [20]'s Scales of Psychological Well-being.

\subsection{Validity and Reliability}

Confirmatory factor analysis and Cronbach's $\alpha$ coefficient were used to verify the validity and reliability of the measurement. Confirmatory factor analysis is a validity test method that analyzes how much a measurement factor explains the concept of a potential factor. It involves using a certain procedure to check whether it is applicable to analyzing the collected data [22]. Regarding the fitness index of each study variable, the fitness indices of the leisure facilitation factor were 2.487 for $\mathrm{x}^{2} / \mathrm{df}, .910$ for TLI, .936 for CFI, and .065 for RMSEA. The fitness indices of the psychological well-being factor were 2.968 for $\mathrm{x}^{2} / \mathrm{df}, .931$ for TLI, .943 for CFI, and .075 for RMSEA. Therefore, it can be said that the validity of the questionnaire items to be verified in this study has been sufficiently secured.

To verify the reliability of this study, Cronbach's $\alpha$ coefficient was calculated; if the reliability index is over .6, it can be said that the reliability is secured [28]. The reliability coefficients of leisure facilitation, which is a variable in this study, were .691, .683, and .798, and the reliability coefficient of overall leisure promotion was .831 . The reliability coefficients of psychological well-being were $.957, .881, .898$, and .832 , and the reliability coefficient of overall psychological well-being was .963 . 


\subsection{Data Proceeding}

A questionnaire was used as the research tool in this study. The survey was conducted from June 2020 to December 2020 while targeting adult men and women participating in golf activities who lived in Seoul, Incheon, and Gyeonggi Province. It was specifically limited to adult men and women in each region who had participated in golf activities for more than 6 months, and a survey was conducted with a focus on golf driving ranges.

\subsection{Ethics statement}

All of the study procedures were reviewed and approved by the Institutional Ethics Review Board of the Sports Research Ethics Committee and conducted according to the principles expressed in the Declaration of Helsinki. After being provided with explanations of the purposes and length of this research study, all of the participants provided consent to participate. They understood that they could refuse to participate in this research study at any time. The participants agreed to allow researchers to use their personal information, which was obtained from questionnaires, for the aim of this study.

\section{Results}

\subsection{Correlation}

The results of the correlation analysis between leisure facilitation and psychological wellbeing, the variables examined in this study. The results showed a significant correlation at the significance level of 0.05 between the sub-factors of leisure facilitation and psychological wellbeing. The personal factors of leisure facilitation were found to be correlated with the intrinsic factors of psychological well-being $(\mathrm{r}=.474)$, flow $(\mathrm{r}=.456)$, self-realization $(\mathrm{r}=.468)$, and confidence $(\mathrm{r}=.517)$. The interpersonal factors were found to be positively correlated with intrinsic factors $(\mathrm{r}=.182)$, flow $(\mathrm{r}=.232)$, self-realization $(\mathrm{r}=.185)$, and confidence $(\mathrm{r}=.309)$. The structural factors were found to be correlated with intrinsic factors $(\mathrm{r}=.258)$, flow $(\mathrm{r}=.253)$, selfrealization ( $\mathrm{r}=.296)$, and confidence $(\mathrm{r}=.254)$. On the other hand, there is no multi-collinearity problem, because the relationship between the research variables multi-collinearity does not exceed the standard of 0.80 , meaning the research could continue.

\subsection{Multiple regression analysis of the effect of leisure facilitation on psychological well- being}

\subsubsection{The effect of leisure facilitation on the intrinsic of psychological well-being}

The results of the multiple regression analysis of the effect on leisure facilitation and psychological well-being are listed in [Table 1]. First, $22.8 \%$ of the intrinsic factors were explained by leisure facilitation with $\mathrm{R}^{2}=.228$, and the regression equation was found to be significant at the significance level of .05 with the $F$ value $=35.556(\mathrm{p}<.001)$. Among the leisure facilitation factors, the B (regression coefficient) of personal facilitation was found to be .592 , and $\mathrm{t}=8.698$, which showed a statistically significant effect at the significance level of .05 $(\mathrm{p}<.05)$. The B (regression coefficient) of structural facilitation was .117 , and $\mathrm{t}=2.032$, which indicated a statistically significant effect at the significance level of .05 ( $\mathrm{p}<.05)$. Regarding the relative contribution of leisure facilitation to psychological well-being, it followed personal facilitation $(\beta=.442)$ and structural facilitation $(\beta=.119)$. However, interpersonal promotion did not affect the intrinsic factors. 
Table 1. Results of multiple regression analysis of influence of leisure facilitation on intrinsic

\begin{tabular}{|c|c|c|c|c|}
\hline & $\mathrm{B}$ & Std E & $\beta$ & $\mathrm{t}$ \\
\hline Constant & 1.360 & .285 & & $4.768 * * *$ \\
\hline Personal & 592 & .068 & .442 & $8.698 * * *$ \\
\hline Interpersonal & -.033 & .066 & -.029 & -.506 \\
\hline Structural & .117 & .057 & .119 & $2.032^{*}$ \\
\hline
\end{tabular}

\subsubsection{The effect of leisure facilitation on the flow of psychological well-being}

The results of a multiple regression analysis of the effect on the leisure facilitation and psychological well-being results are shown in [Table 2]. First, $21.3 \%$ of the flow was explained by leisure facilitation with $\mathrm{R}^{2}=.213$, and the regression equation was found to be statistically significant at the significance level of .05 with the $F$ value $=32.670(\mathrm{p}<.001)$. Among the leisure facilitation factors, the B (regression coefficient) of personal facilitation was .502, and $t=8.014$, which was statistically significant at the significance level of $.05(\mathrm{p}<.001)$. However, interpersonal and structural promotion did not affect the flow.

Table 2. Results of multiple regression analysis of influence of leisure facilitation on flow

\begin{tabular}{|c|c|c|c|c|}
\hline & $\mathrm{B}$ & Std E & $\beta$ & $\mathrm{t}$ \\
\hline Constant & 1.404 & .263 & & $5.344 * * *$ \\
\hline Personal & .502 & .063 & .411 & $8.014 * * *$ \\
\hline Interpersonal & .058 & .061 & .055 & .946 \\
\hline Structural & .069 & .053 & .077 & 1.309 \\
\hline$R^{2}=.213, F=32.670^{* * *}, * * * p<.001$ &
\end{tabular}

\subsubsection{The effect of leisure facilitation on the self-realization aspect of psychological well- being}

The results of the multiple regression analysis of the effect of leisure promotion on psychological well-being are listed in [Table 3]. First, $23.4 \%$ of self-realization was explained by leisure facilitation with $\mathrm{R}^{2}=.234$, and the regression equation was significant at the significance level of .05 with $\mathrm{F}$-value $=36.758(\mathrm{p}<.001)$. Among the leisure facilitation factors, the B (regression coefficient) of personal facilitation was .590, and $t=8.364$ was found to have a statistically significant effect at the significance level of $.05(\mathrm{p}<.05)$. The B (regression coefficient) of structural facilitation was .181 , and $t=3.037$, which was found to have a statistically significant effect at the significance level of .05 $(\mathrm{p}<.05)$. Regarding the relative contribution of leisure facilitation to psychological well-being, it followed personal facilitation $(\beta=.423)$ and structure facilitation $(\beta=.119)$. However, interpersonal facilitation did not affect self-realization. 
Table 3. Results of multiple regression analysis of influence of leisure facilitation on self-realization

\begin{tabular}{|c|c|c|c|c|}
\hline & $\mathrm{B}$ & Std E & $\beta$ & $\mathrm{t}$ \\
\hline Constant & 1.016 & .295 & & $3.440^{* * *}$ \\
\hline Personal & .590 & .071 & .423 & $8.364 * * *$ \\
\hline Interpersonal & -.062 & .068 & -.052 & -.910 \\
\hline Structural & .181 & .059 & .176 & $3.037 * *$ \\
\hline
\end{tabular}

$R^{2}=.234, F=36.758 * * *, * * p<.01, * * * p<.001$

\subsubsection{The effect of leisure facilitation on the confidence aspect of psychological well-being}

The results of the multiple regression analysis of the effect of leisure facilitation on psychological well-being are listed in [Table 4]. First, leisure facilitation explained $28.3 \%$ of confidence with $\mathrm{R}^{2}=.283$, and the regression equation was statistically significant at the significance level of .05 with F-value $=47.226(\mathrm{p}<.001)$. Among the leisure facilitation factors, the $B$ (regression coefficient) of personal facilitation was .720 , and $t=9.511$, which was statistically significant at the significance level of .05 ( $\mathrm{p}<.001)$. The B (regression coefficient) of interpersonal facilitation was found to have a statistically significant effect at the significance level of .05 with $t=2.820$ ( $p<.01)$. Regarding the relative contribution of leisure facilitation to psychological well-being, it followed personal facilitation $(\beta=.465)$ and interpersonal facilitation $(\beta=.157)$. However, structural facilitation did not affect the confidence aspect of psychological well-being.

Table 4. Results of multiple regression analysis of influence of leisure facilitation on confidence

\begin{tabular}{|c|c|c|c|c|}
\hline & B & Std E & $\beta$ & $\mathrm{t}$ \\
\hline Constant & .307 & .317 & & .968 \\
\hline Personal & .720 & .076 & .465 & $9.511^{* * *}$ \\
\hline Interpersonal & .207 & .073 & .157 & $2.820^{* *}$ \\
\hline Structural & .001 & .064 & .001 & .021 \\
\hline
\end{tabular}

$R^{2}=.283, F=47.226^{* * *}, * * * p<.001$

\section{Discussion}

First, the personal and structural factors of leisure facilitation among amateur golfers affected the intrinsic factors of psychological well-being. In the leisure activity of golf, it is judged that personal factors such as individual interest or motivation have a positive influence on intrinsic psychological well-being, such as satisfaction and happiness. According to Woo, Lee, and Kang [29], personal facilitation among the sub-factors of leisure facilitation of badminton hobbyists had a significant effect on the 'patience' aspect of serious leisure. Kang,

Second, the personal factors of leisure facilitation among amateur golfers affected the flow factors of psychological well-being. In general, a golfer uses 14 different clubs. As golfers use a variety of golf clubs, feeling a good fit with each club helps one experience strong immersion 
and psychological well-being. Kim [34] reported that participants in life sports experience immersion while participating in sports, and that through this immersion experience, they can feel psychological happiness as they experience goal achievement, a sense of achievement, and stress relief

Third, the leisure facilitating factors of the personal and structural factors of Korean amateur golfers have been shown to affect the self-actualization sense of psychological well-being. Golf is a mental sport, and personal factors are very important; among leisure facilitation, these include joy, satisfaction, strong pleasure, and happiness. When participating in the sport of golf, among personal facilitation factors, it appears that there are high self-realization factors of psychological well-being. The results of Lee [37] indicate that individuals most value leisure activities when there are a wide variety of programs and facilities available.

Fourth, regarding the individual factors of leisure facilitation among Korea's amateur golfers, human factors affected the confidence of psychological well-being. It is an individual sport, but it takes four people to have a full group for a round of golf. Therefore, it is judged that interpersonal facilitation factors have a positive effect on psychological well-being. Lee [41] said that the motivation to feel a sense of belonging while interacting with other people and the continuous leisure experience are helpful to one's daily life, and that life maintains psychological flow.

\section{Conclusion}

This study aims to investigate the relationship between leisure facilitation and psychological well-being among Korean amateur golfers. The participants of this study were adult men and women who participate in golf activities and who live in Seoul, Incheon, and Gyeonggi-do. For the data analysis in this study, frequency, confirmatory factor, reliability, correlation, and multiple regression analyses were performed. The results were as follows. First, personal factors and structural factors of leisure facilitation affected the intrinsic factors of psychological well-being. Second, the individual factors of leisure facilitation affected the intrinsic factors of psychological well-being. Third, the individual and structural factors of leisure facilitation affected the self-realization of psychological well-being. Fourth, the individual factors of leisure facilitation affected the confidence of psychological well-being. So, we found that it is necessary to build an infrastructure to participate in golf as a leisure activity, and the high psychological well-being had an effect on improving the quality of life of individuals.

We would suggest for follow-up research are as follow. First, this study was conducted only for residents of Seoul, Incheon, and Gyeonggi-do, so it is limited to represent amateur golfers in South Korea. Second, this study was conducted quantitatively. It is necessary to study through qualitative research on leisure facilitation and psychological well-being, which could not be revealed by quantitative research.

\section{Acknowledgements}

This Paper was supported by the Sehan University Research Fund in 2021.

\section{References}

[1] Korea Culture and Tourism Institute, "2008 a white paper on leisure," (2009)

[2] L. A. Raymore, "Facilitators to leisure," Journal of Leisure Research, vol.34, no.1, pp.37-51, (2002)

[3] B. Kim and Y. Lee, "The relationship among leisure facilitator, commitment and intention to continue of zumba participants,” The Korean Journal of Physical Education, vol.57, no.1, pp.291-304, (2018) 
[4] S. Bock and H. Kim, "The causal relationship analysis among leisure facilitators, leisure constraints negotiation, serious leisure for winter sports participants," The Korean Journal of Physical Education, vol.55, no.1, pp.579588, (2016)

[5] F. M. Andrew and S. B. Withey, "Social indicators of well-being," New York and London, Plenum, pp.20-31, (1976)

[6] Campbell, "Subjective measures of well-being," American Psychologist, vol.31, no.2, 117-124, (1976)

[7] Y. Kim and H. Lee, "A study of developing the leisure participation condition type for integrating leisure constraints and facilitators," The Korea Academic Society of Tourism and Leisure, vol.23, no.2, pp.261-277, (2011)

[8] S. Waterman, "Two conception of happiness; Contrasts of personal expressiveness (eudaimonia) and hedonic enjoyment," Journal of Personality and Social Psychology, vol.64, pp.678-691, (1993)

[9] D. Ryff, "Happiness is everything, or is it? Explorations on the meaning of psychological well-being," Journal of Personality and Social Psychology, vol.57, pp.1069-1081, (1989)

[10] S. Hong, "The criteria for selecting appropriate fit indices in structural equation modeling and their rationales," Korean Journal of Clinical Psychology, vol.19, no.1, pp.161-177, (2000)

[11] T. Sung, "Theory and practice of item creation and analysis," Hakjisa, (2006)

[12] J. Woo, C. Lee, and H. Kang, "Relationship between leisure facilitators and serious leisure among badminton club members," Journal of Leisure Studies, vol.18, no.2, pp.1-20, (2020)

[13] S. Kim, "A study on the relationship between exercise commitment, psychological happiness, and exercise addiction of participants in living sports," Journal of Korea Sports Research, vol.17, no.6, pp.451-460, (2006)

[14] S. Lee, "The moderating effect of leisure constraint negotiation on the relationship between leisure facilitator and recreation specialization," The Korean Journal of Physical Education, vol.56, no.5, pp.537-548, (2017)

[15] B. Lee, "Relationship of participation motivation in running, self-efficacy, serious leisure and psychological commitment," Journal of Tourism Sciences, vol.39, no.7, pp.53-69, (2015) 
A Study on Social Psychology Factors of Golfers in South Korea

This page is empty by intention. 